\title{
ACE I/D AND ACE2 RECEPTOR GENE (RS2106809, RS2285666) POLYMORPHISMS IS NOT RELATED TO THE CLINICAL COURSE OF COVID-19; A CASE STUDY
}

Sevim Karakaş Çelik ${ }^{1}$, Güneş Çakmak Genç ${ }^{1}$, Nihal Piskin ${ }^{1}$, Bilgehan Acikgoz ${ }^{2}$, Bülent Altınsoy $^{1}$, Başak Kurucu İsssiz ${ }^{1}$, and Ahmet Dursun ${ }^{1}$

${ }^{1}$ Zonguldak Bülent Ecevit Üniversitesi Tıp Fakültesi

${ }^{2}$ Zonguldak Bülent Ecevit University

March 14, 2021

\begin{abstract}
Objectives: Coronavirus disease 2019 (COVID-19) is an infectious disease, that's the reason for the currently ongoing pandemic, caused by severe acute respiratory syndrome coronavirus 2 (SARS-CoV-2). Angiotensin-converting enzyme (ACE2) has been recognized as the specific receptor of the SARS-CoV-2 virus. Although the possible effect of ACE2 gene polymorphism remains unknown, human ACE2 receptor expression influences SARS-CoV-2 susceptibility and COVID-19 disease outcome. In this study, we aimed to investigate the relationship between ACE gene I/D polymorphism, ACE2 receptor gene polymorphism, and Covid-19 severity. Methods: ACE gene I/D polymorphism and ACE2 receptor gene rs2106809 and rs2285666 polymorphisms were determined using PCR and PCR-RFLP methods, respectively in 155 Covid19 patients who were divided into 3 groups (mild, moderate, and severe) according to clinical symptoms. Results: However, the distribution of genotype and allele frequencies of ACE gene I/D, ACE2 receptor gene rs2106809, and rs2285666 polymorphisms was not statistically significant in all groups. Conclusions: In conclusion, in the study population, ACE gene I/D, ACE2 receptor gene rs2106809, and rs2285666 polymorphisms were not associated with the severity of Covid-19 infection. Although, ACE2 receptor gene expression may affect the susceptibility to Covid-19, there is no existing evidence that the ACE or ACE2 gene polymorphisms are directly associated with Covid-19 severity. Interindividual differences in covid-19 severity might be related to epigenetic mechanisms of ACE2 receptor gene expression or variations in other genes suggested to have critical role in COVID-19 pathogenesis such as proinflammatory cytokines, coagulation indicators. Keywords: COVID-19, ACE , ACE2, rs2106809, rs2285666
\end{abstract}

ACE I/D AND ACE2 RECEPTOR GENE (RS2106809, RS2285666) POLYMORPHISMS IS NOT RELATED TO THE CLINICAL COURSE OF COVID-19; A CASE STUDY

Sevim KARAKAŞ ÇELIK ${ }^{1}$, Güneş ÇAKMAK GENÇ${ }^{1}$, Nihal Pİ̧KKIN² ${ }^{2}$, Bilgehan AÇIKGÖZ³ , Bülent ALTINSOY $^{4}$, Başak KURUCU IŞSiziz ${ }^{1}$, Ahmet DURSUN ${ }^{1}$

${ }^{1}$ Department of Medical Genetics, Zonguldak Bulent Ecevit University, Zonguldak, Turkey

${ }^{2}$ Department of Infectious Diseases and Clinical Microbiolog, Zonguldak Bulent Ecevit University, Zonguldak, Turkey

${ }^{3}$ Department of Public Health, Zonguldak Bulent Ecevit University, Zonguldak, Turkey

${ }^{4}$ Department of Pulmonary Medicine, Zonguldak Bulent Ecevit University, Zonguldak, Turkey

Corresponding Address:

Dr. Sevim KARAKAŞ ÇELIKK 
Bülent Ecevit Üniversitesi

Tıp Fakültesi, Tıbbi Genetik Anabilim Dalı

ZONGULDAK

Phone: +90372 2613221

E mail: sevimkarakas@hotmail.com

\section{Abstract}

Objectives: Coronavirus disease 2019 (COVID-19) is an infectious disease, that's the reason for the currently ongoing pandemic, caused by severe acute respiratory syndrome coronavirus 2 (SARS-CoV-2). Angiotensin-converting enzyme (ACE2) has been recognized as the specific receptor of the SARS-CoV-2 virus. Although the possible effect of ACE2 gene polymorphism remains unknown, human ACE2 receptor expression influences SARS-CoV-2 susceptibility and COVID-19 disease outcome. In this study, we aimed to investigate the relationship between ACE gene I/D polymorphism, ACE2 receptor gene polymorphism, and Covid-19 severity.

Methods: ACE gene I/D polymorphism and ACE2 receptor gene rs2106809 and rs2285666 polymorphisms were determined using PCR and PCR-RFLP methods, respectively in 155 Covid19 patients who were divided into 3 groups (mild, moderate, and severe) according to clinical symptoms.

Results: However, the distribution of genotype and allele frequencies of ACE gene I/D, ACE2 receptor gene rs2106809, and rs2285666 polymorphisms was not statistically significant in all groups.

Conclusions: In conclusion, in the study population, ACE gene I/D, ACE2 receptor gene rs2106809, and rs2285666 polymorphisms were not associated with the severity of Covid-19 infection. Although, ACE2 receptor gene expression may affect the susceptibility to Covid-19, there is no existing evidence that the ACE or ACE2 gene polymorphisms are directly associated with Covid-19 severity. Interindividual differences in covid-19 severity might be related to epigenetic mechanisms of ACE2 receptor gene expression or variations in other genes suggested to have critical role in COVID-19 pathogenesis such as proinflammatory cytokines, coagulation indicators.

Keywords: COVID-19, ACE , ACE2, rs2106809, rs2285666

\section{What's already known about this topic?}

The relationship between ACE2 gene expression and Covid-19 has been shown in the literature, however, the studies conducted to investigate the role of $\mathrm{ACE}$ receptor gene polymorphisms are in-silico analyzes or epidemiological studies.

\section{What does this article add?}

There is no study investigating the relationship between ACE I/D and ACE2 receptor gene rs2106809 and rs2285666 polymorphism and Covid-19 severity. This study showed that ACE gene I/D, ACE2 receptor gene rs2106809, and rs2285666 polymorphisms were not associated with the severity of Covid-19 infection.

\section{Introduction}

Coronaviruses are a large family of single-stranded RNA viruses, which cause illnesses ranging from mild cold to more severe diseases such as Severe Acute Respiratory Syndrome (SARS)-CoV and Middle East Respiratory Syndrome (MERS-CoV) [1]. COVID-19 was first reported in Wuhan, China, and subsequently spread worldwide. As of February 2021, there have been more than a hundred million cases of COVID-19 worldwide and nearly two and a half million deaths [2].

The entry of SARS-CoV-2, the agent that causes COVID-19, into the cell occurs by binding viral spike proteins to Angiotensin-Converting Enzyme 2 (ACE2) receptors of the host membrane. It was suggested that increase susceptibility to Covid-19 infection is associated with the expression of the target ACE2 receptor 
in the epithelium exposed to the virus [3]. The low infection and complication level is a fact among children. Serum ACE levels in children are higher than in adults and ACE2 receptor gene expression in the nasal epithelium that is the first point of contact for SARS-CoV-2, was age-dependent, lowest in younger children and increasing with age into adulthood [4]. It was also suggested that the lower risk among children is due to the lower expression of the ACE2 receptor [5]. Epidemiological data show that a majority of pediatric COVID-19 cases showed mild to moderate clinical features, and only a few developed severe or critical diseases (0.6\% and $0.3 \%$, respectively) [6]. Total 44672 cases, only $1 \%$ is under 10 years old and in China [7]. Also lower ACE2 receptor expression in children, highlighting the importance of ACE and ACE2 receptor expression level in Covid19 severity.

A counter-regulatory relationship between ACE2 and ACE located on opposite axes in the Renin-Angiotensin System (RAS) was reported. ACE plays important role in converting angiotensin I to angiotensin II and $\mathrm{ACE} 2$ is a negative regulator of the RAS and counterbalances the function of ACE. The lungs are primary organs for ACE receptor expression and generating circulating angiotensin II. RAS plays a role in the pathogenesis of pulmonary hypertension and fibrosis, which are common chronic lung diseases. Recent studies show that RAS also plays important role in acute lung diseases, particularly acute respiratory distress syndrome (ARDS) [8].

A common 287 base pair insertion/deletion (I/D) polymorphism has been reported in intron 16 of the ACE gene and is known to be associated with serum levels of circulating ACE. Serum ACE concentrations were reported to be significantly higher in subjects with the $\mathrm{D} / \mathrm{D}$ genotype compared to the $\mathrm{I} / \mathrm{D}$ and $\mathrm{I} / \mathrm{I}$ genotypes [9]. Considering the opposite effect between ACE and ACE2, decreased ACE2 receptor gene expression is strongly related to an increase in ACE expression. So it could be hypothesized that having a D allele for ACE I/D polymorphism affects the clinical course of the Covid-19 by decreasing the ACE2 receptor level.

Besides, it has been shown that patients with chronic obstructive pulmonary disease (COPD) have increased gene expression of ACE2 receptor in bronchial epithelial cells in the lower respiratory tract, and also smoking has been shown to increase both the expression and activity of ACE-2 in the airways. While up-regulation of ACE-2 is beneficial in protecting the host against acute lung injury, this makes individuals more susceptible to coronavirus infections that use this receptor to enter epithelial cells $[10,11]$.

In the changes in ACE2 receptor gene expression level, genetic variations play a very important role as well as environmental factors. Of the many polymorphisms identified in the ACE2 receptor gene, rs2106809 and rs2285666 are particularly remarkable. A bioinformatics tool called Human Splicing Finder (HSF) (v.3.0) predicts that rs2106809 polymorphism, intronic SNP found in the ACE2 receptor gene, might create an intronic-exonic splicing enhancer site (ESE). It was suggested that the splicing efficiency of the ACE2 receptor gene may be influenced by the creation of these enhancer motifs [12]. It was also found that ACE2 rs2106809 CC or CT genotype carriers had higher circulating ACE2 receptor levels compared to TT genotype carriers [13].

G8790A (rs2285666) polymorphism is at the fourth base of the third intron and situated in the intron adjoined to the exon, suggesting, this locus could alter mRNA alternate splicing and affect ACE2 receptor gene expression. It has also been reported that this polymorphism shows a strong linkage disequilibrium with the other SNPs (rs1978124 intron 1 and rs714205 intron 16) in the ACE2 receptor gene [14].

The relationship between ACE2 gene expression and Covid-19 has been shown in the literature, however, most of the studies conducted to investigate the role of ACE receptor gene polymorphisms are in-silico analyzes or epidemiological studies. Also, to the best of our knowledge, there is no study investigating the relationship between ACE I/D and ACE2 receptor gene rs2106809 and rs2285666 polymorphism and Covid19 severity. In this study, it is aimed to investigate whether the course of the disease (mild, moderate, and severe) is predictable by determining the genotypes of ACE I/D and ACE2 receptor gene polymorphism.

\section{Materials and methods}

One hundred fifty-five Covid-19 patients were included in this study. A confirmed case with COVID-19 was 
defined as a positive result to real-time reverse-transcriptase polymerase-chain-reaction (RT-PCR) assay for nasal and pharyngeal swab specimens [15]. Written informed consent was obtained from all the patients that participated in this study, and the local ethics committee of Bulent Ecevit University, Faculty of Medicine approved the study. Patients with Covid-19 were grouped according to the severity of the disease. Clinical characteristics of patients are shown in Table 1. COVID-19 severity is classified as follows; Mild type: Outpatients, the clinical symptoms are mild, with no abnormal radiological findings, Moderate type: Hospitalized for persistent fever or pneumonia or mild respiratory distress or present pneumonia on chest CT, Severe type: The disease is classified as severe if one of the following conditions is met:

- Respiratory distress, respiratory rate [?]30/min.

- Oxygen saturation on room air at rest [?]93\%.

- The partial pressure of oxygen in arterial blood/FiO2 [?]300 mm Hg.

- Respiratory failure occurs and mechanical ventilation is required.

- Another organ dysfunction is present, requiring ICU monitoring and treatment.

\section{DNA isolation and Genotyping}

Genomic DNA was extracted from $200 \mu$ l of peripheral blood by Macherey-Nagel DNA isolation Kit (Cat No: 740.951.250) following manufacturer instructions.

Polymerase chain reaction (PCR) was used to detect the I/D polymorphism of the ACE gene. A polymerase chain reaction (PCR)-based restriction fragment-length polymorphism (RFLP) method was used to genotype ACE2 rs2106809 and rs2285666 polymorphisms. The PCR was performed in a $25 \mu \mathrm{l}$ volume for each polymorphism that is containing 10X PCR buffer, $3.0 \mathrm{mM} \mathrm{MgCl} 2,0.25 \mathrm{mMdNTPs}, 1.5$ units of Taq polymerase (Promega, Madison, WI), and $0.3 \mu \mathrm{M}$ each primers (5' CTG GAG ACC ACT CCC ATC CTT TCT 3' and 5' GAT GTG GCC ATC ACA TTC GTC AGA 3' for ACE I/D polymorphism and F; 5'-GAA AGC CAG ATG CTT TAA CAA G-3' and R; 5'-TTTTTCCATATCTCTATCTGATCG-3' for ACE2 rs2106809 polymorphism and F; 5'-CATGTGGTCAAAAGGATATC-3' R; 5'- AAAGTAAGGTTGGCAGACAT-3' for ACE2 rs2285666 polymorphism.

For ACE I/D polymorphism the PCR products were run at $2 \%$ agarose gel. Allele (I/D) types and genotype for each sample were determined based on the PCR product sizes. The major allele indicates the $490 \mathrm{bp}$ fragment that embraces the $287 \mathrm{bp}$ Alu sequence, known as an insertion, whereas the $190 \mathrm{bp}$ fragment represents the minor allele with the deletion of the Alu sequence. Heterozygosity specifies the combination of both major and minor fragments i.e. 490/190 bp.

For ACE2 rs2106809 polymorphism; The PCR products of 207 base pairs (bp) were digested with restriction enzyme TaqI resulting in two products of $183 \mathrm{bp}$ and $24 \mathrm{bp}$ in the presence of A allele.

For ACE2 rs2285666 polymorphism; The PCR products were incubated with $5 \mathrm{U}$ of AluI restriction enzyme at overnight. After digestion, fragments of 281 and 185 bp identify T allele and a 466 bp band identify C allele.

All digestion products were electrophoresed on 3\% agarose gel and visualized by staining with ethidium bromide and evaluated using the gel documentation system (Syngene, Genegenius Bio Imaging System).

\section{Statistical analysis}




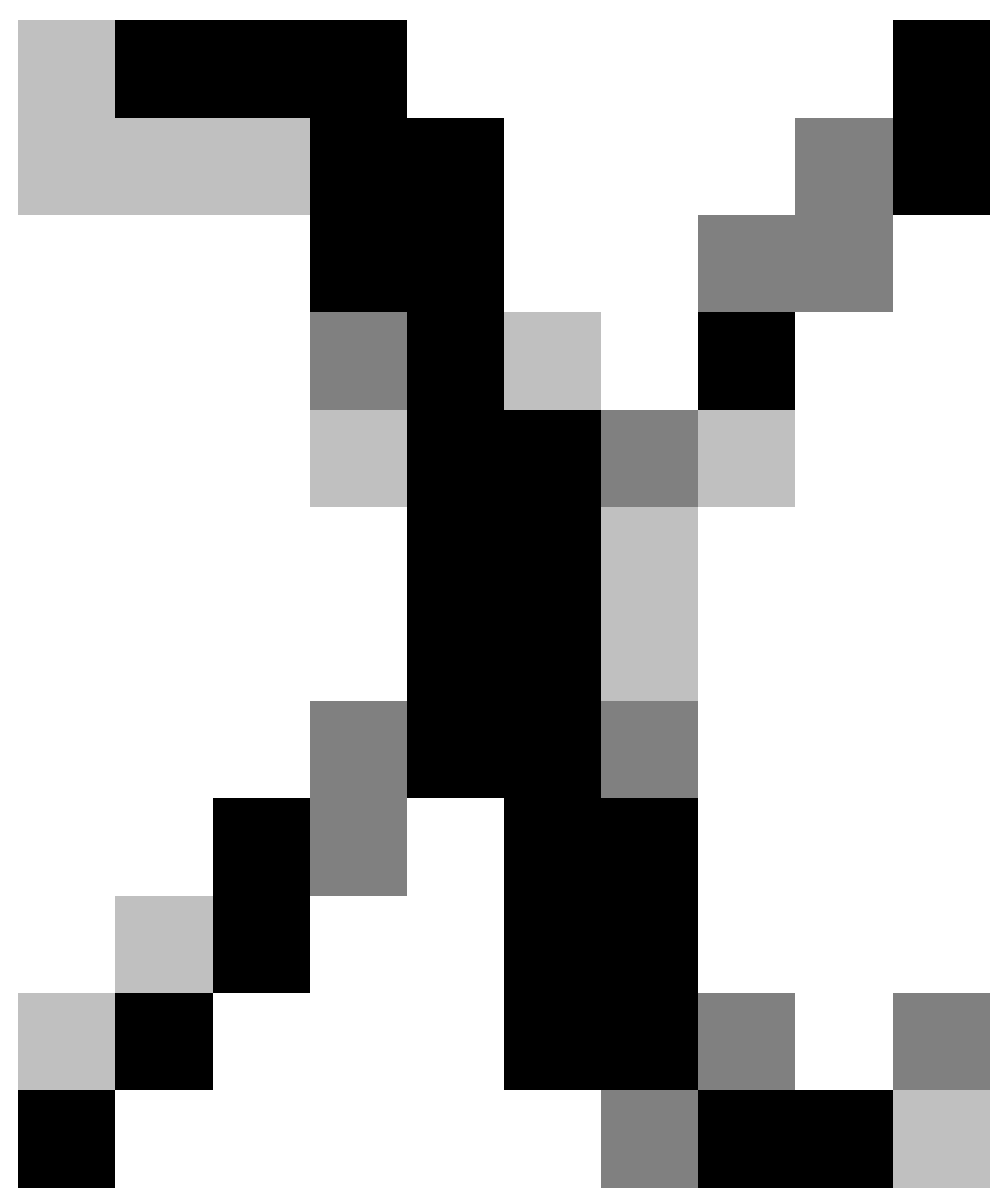

Genotype and allele frequency of the polymorphism was calculated in cases according to the severity of Covid19. The ${ }^{2}$ test was used to compare genotype frequency of the ACE and ACE2 receptor gene polymorphisms in patients who have mild, moderate, or severe clinical symptoms. Because the ACE2 receptor gene is located on the X chromosome and as males are hemizygous for ACE2, males and females were analyzed separately. A p-value less than 0.05 was considered statistically significant. The software used for the calculations was SPSS version 18 (SPSS Inc., Chicago, IL).

\section{Results}

The present study included 155 Covid19 patients of which 78 are in the mild, 42 are in the moderate and 35 are in the severe group. Seventy-seven subjects were women and 78 were men enrolled in the study. The mean $( \pm \mathrm{SD})$ age was $52.25 \pm 17.52$ in patients, and $44.60 \pm 15.52,54.57 \pm 16.62$, and $66.54 \pm 12.68$ for mild, moderate, and severe groups, respectively. The severity of the disease has increased with age $(\mathrm{p}<0.001)$. Also, it was found that gender differences affect covid19 severity and men tended to be more serious than women $(\mathrm{p}=0.003)$. The percentage of males was $60 \%$ in severe groups and that is $40 \%$ for females. The clinical signs and symptoms of the Covid19 patients are given in Table 1.

The distribution of each genotype for ACE I/D, ACE2 rs2106809, and rs2285666 in the Covid19 patients 
according to the severity of disease are shown in Table 2. There was no statistically significant association between groups according to genotype $(\mathrm{p}=0.191)$ and allele frequencies $(\mathrm{p}=0.392)$ for $A C E I / D$ gene polymorphism.

Because ACE2 is on the $\mathrm{X}$ chromosome, data for each sex were analyzed separately in Table 2. It was also found that the distribution of genotype and allele frequencies of ACE2 rs2106809 and rs2285666 gene polymorphisms was similar in all groups according to the severity of disease for both genders $(\mathrm{p}>0.05)$. Haplotype frequency and association analysis were also performed (Table 3). We identified one haplotype for two polymorphisms (rs2106809 and rs2285666) in the ACE2 receptor gene. However, no statistical difference was noted $(\mathrm{p}=0.519)$.

Also genotypic and allelic distribution of ACE I/D and ACE2 rs2106809 and rs2285666 polymorphisms were analyzed according to clinical symptoms and no significant relationship was found.

\section{Discussion}

ACE2 has been established as the functional host receptor for SARS-CoV-2, responsible for the current devastating worldwide pandemic of coronavirus disease 2019 (COVID-19) [16]. COVID-19 displays symptoms ranging from a mild cold to cardio-respiratory failure that may lead to death $[17,18]$. Both ACE and ACE2 receptor genes have numerous genetic variations including functional polymorphism of insertion/deletion (Ins/Del) for ACE and rs2106809 and rs2285666 polymorphisms for ACE2 receptor gene. The del allele shows higher ACE activity and polymorphisms in the ACE2 receptor gene affect circulating ACE2 receptor levels $[12,13]$. Considering the role of ACE in Covid-19 pathogenesis and the variation in disease severity, ACE I / D and ACE2 receptor gene variants have attracted the attention of researchers. However, the studies were limited to in silico analyzes and epidemiological studies. To the best of our knowledge, our work is the first study including wet lab analysis for investigating ACE I/D, ACE2 rs2106809, rs2285666 polymorphisms. Unfortunately, no association was found between these polymorphisms and the severity of symptoms of Covid-19.

Delanghe et all conducted a multiple regression analysis model to compare the prevalence and mortality of the COVID-19 infection from a number of European countries and frequency data of the geographical variation of $\mathrm{I} / \mathrm{D}$ polymorphism in the $A C E$ gene. Differently, the authors reported a negative correlation between the frequency of the $\mathrm{D}$ allele of the $A C E$ Ins/Del polymorphism and prevalence and mortality rates of COVID-19 in 33 countries. It should be noted that this study does not involve wet lab analysis and the results conflicted with eastern Asian populations data. It was suggested a converse association between ACE D allele frequency and prevalence of Covid-19. Considering that the D allele frequency is lower in Asian populations than in European populations, the mortality rate and prevalence of Covid-19 in the Asia population is expected to be higher. However, the prevalence and mortality of COVID-19 in Europe are higher than in Asia [19,20]. On the contrary to Delangheet all, Pati et al. suggested a significant positive correlation of D allele of ACE polymorphism with SARS-CoV-2 infection and mortality rate in their epidemiological investigation [21].

Hatami et al. conducted a meta-analysis study and showed the recovery rate significantly increased with the I/D allele frequency ratio. However, this study provides an ecological perspective but does not provide a direct clinical relevance between the COVID-19 and ACE I/D polymorphism [22].

In two different populations, genetic variants in the ACE2 receptor gene were analyzed by whole-exome sequencing in hospitalized COVID-19 patients. Differently from our study, they investigated the relation of Covid19 with coding-region variants in the ACE2 receptor gene. These studies also provided no strong evidence that ACE2 receptor gene variants are a consistent association with COVID-19 severity. The ACE2 receptor gene sequencing showed no coding sequence variants that could explain an increased risk of developing COVID-19. Similarly, in our study, no relationship was found between ACE-2 receptor gene intron variants and Covid19 severity [23,24].

Our results showed that ACE I/D, ACE2 rs2106809, rs2285666 polymorphisms have no role in the severity 
of Covid-19. Novelli et al. (2020) also reported that ACE2 receptor gene coding-region variants have no effects on covid-19 severity [23]. Taking that ACE2 receptor gene expression may affect the susceptibility of individuals to infection into consideration, we speculate that genetic variations in the non-coding regions of the ACE2 receptor gene or in other non-coding DNAs that control the expression levels of ACE genes may have a potential role in the severity of the disease.

A growing number of recent findings point out that epigenetic mechanisms such as DNA methylation and histone modifications, play key roles to control gene expression. A recent analysis of public genomic and transcriptomic data outlined the role of histone methylation to regulate ACE2 receptor gene transcription [25]. Further regulation occurs at the mRNA level. From putative microRNA-binding sites identified in vitro, Lambert et al. (2014) demonstrated that miR-421 downregulates the ACE2 receptor gene [26]. Besides undergoing post-translational modifications by glycosylation and phosphorylation, the ACE2 receptor gene is also post-translationally regulated [27]. Therefore, variations in other genes related to epigenetic mechanisms of ACE2 receptor gene expression may have a potential role in the severity of Covid-19.

Second, mortality or severity of disease in COVID-19 patients might be linked to excessive production of proinflammatory cytokines leads to ARDS aggravation and widespread tissue damage resulting in multiorgan failure and death, not linked to increased ACE2 receptor expression. Wang et al. showed that most of the severely ill patients had viral shedding in a variety of tissues for 20-40 days after onset of disease, while the majority of mildly ill patients had viral shedding restricted to the respiratory tract and had no detectable virus RNA 10 days after onset [28]. Similarly, Liu et al. (2020) reported that mild cases were found to have an early viral clearance [29]. These results may indicate that the nasopharyngeal SARS-CoV-2 RNA load is higher in the severe group, or they may be related to the immune response of the host. In the current literature impairment of SARS-CoV-2 clearance is related due to genetic and viral features, enhanced levels of interferons, neutrophil extracellular traps and pyroptosis, and possible other unknown mechanisms were reported [30]. Besides, the patients from the severe group showed elevated levels of inflammatory cytokines (such as IL-2, IL-2R, RIL-6, IL-8, and IL-10), significant enhancement in coagulation parameters (such as D-dimer, prothrombin time, and fibrinogen), and increases in myocardial injury indicators. Therefore, inflammatory release, coagulation dysfunction, and myocardial injury correlate with disease severity and rise throughout the course of the disease [31,32]. Thus, the severity of COVID-19 may be related to variations in genes encoding proinflammatory cytokines or other genes that are suggested to be associated with the occurrence of severe COVID-19.

In conclusion, our study does not support the hypothesis that ACE I/D, ACE2 rs2106809, rs2285666 polymorphisms are related to Covid-19 severity. Considering that little is known about the genetic basis of the difference of Covid-19 severity and analysis of genetic polymorphisms reveals important information, the possible relation between ACE2 rs2106809, rs2285666 polymorphisms, and Covid-19 severity required to be investigated. However, in order to explain the personal variations in Covid-19 pathogenesis, further studies are needed to investigate variations in other genes related to epigenetic mechanisms of ACE2 receptor gene expression and variation in genes encoding proinflammatory cytokines, coagulation indicators.

\section{References}

1. Perlman S, Netland J. Coronaviruses post-SARS: update on replication and pathogenesis. Nat Rev Microbiol 2009;7:439-50.

2. https://www.worldometers.info/coronavirus/ Date last accessed: 09.02.2021

3. Wan Y, Shang J, Graham R, Baric RS, Li F. Receptor recognition by novel coronavirus from Wuhan: an analysis based on decade-long structural studies of SARS. J Virol 2020;94:e00127-20.

4. Rodriguez GE, Shin BC, Abernathy RS, Kendig EL Jr. Serum angiotensin-converting enzyme activity in normal children and in those with sarcoidosis. J Pediatr 1981;99:68-72.

5. Bunyavanich S, Do A, Vicencio A. Nasal Gene Expression of Angiotensin-Converting Enzyme 2 in Children and Adults. JAMA 2020;323:2427-2429. 
6. Guo CX, He L, Yin JY, et al. Epidemiological and clinical features of pediatric COVID-19. BMC Med 2020;18:250.

7. Wu Z, McGoogan JM. Characteristics of and important lessons from the Coronavirus Disease 2019 (COVID-19) Outbreak in China: summary of a report of 72314 cases from the Chinese Center for Disease Control and prevention. JAMA 2020;323:1239-1242.

8. Kuba K, Imai Y, Penninger JM. Angiotensin-converting enzyme 2 in lung diseases. Curr Opin Pharmacol 2006;6:271-6.

9. Rigat B, Hubert C, Alhenc-Gelas F, Cambien F, Corvol P, Soubrier F. An insertion/deletion polymorphism in the angiotensin I-converting enzyme gene accounting for half the variance of serum enzyme levels. J Clin Invest 1990;86:1343-6.

10. Leung JM, Yang CX, Tam A, et al. ACE-2 Expression in the Small Airway Epithelia of Smokers and COPD Patients: Implications for COVID-19. Eur Respir J 2020;55:2000688.

11. Smith JC, Sausville EL, Girish V, et al. Cigarette smoke exposure and inflammatory signaling increase the expression of the SARS-CoV-2 receptor ACE2 in the respiratory tract. Dev Cell 2020;53: 514-529.e3.

12. Chen YY, Zhang P, Zhou XM, et al. Relationship between genetic variants of ACE2 gene and circulating levels of ACE2 and its metabolites. J Clin Pharm Ther 2018;43:189-195.

13. Liu D, Chen Y, Zhang P, et al. Association between circulating levels of ACE2-Ang-(1-7)-MAS axis and ACE2 gene polymorphisms in hypertensive patients. Medicine (Baltimore) 2016;95:e3876.

14. Kramkowski K, Mogielnicki A, Buczko W. The physiological significance of the alternative pathways of angiotensin II production. J Physiol Pharmacol 2006;57:529-539.

15. Huang C, Wang Y, Li X, et al. Clinical features of patients infected with 2019 novel coronavirus in Wuhan, China. Lancet 2020;395:497-506.

16. Zhou P, Yang XL, Wang XG, et al. A pneumonia outbreak associated with a new coronavirus of probable bat origin. Nature 2020;579:270-273.

17. Guan WJ, Ni ZY, Hu Y, et al. Clinical Characteristics of Coronavirus Disease 2019 in China. N Engl J Med 2020;382:1708-1720.

18. Li Q, Guan X, Wu P, et al. Early Transmission Dynamics in Wuhan, China, of Novel CoronavirusInfected Pneumonia. N Engl J Med 2020;382:1199-1207.

19. Delanghe JR, Speeckaert MM, De Buyzere ML. The host's angiotensin-converting enzyme polymorphism may explain epidemiological findings in COVID-19 infections. Clin Chim Acta 2020;505:192-193.

20. Saadat M. No significant correlation between ACE Ins/Del genetic polymorphism and COVID-19 infection. Clin Chem Lab Med 2020;58:1127-1128.

21. Pati A, Mahto H, Padhi S, Panda AK. ACE deletion allele is associated with susceptibility to SARSCoV-2 infection and mortality rate: An epidemiological study in the Asian population. Clin Chim Acta 2020;510:455-458.

22. Hatami N, Ahi S, Sadeghinikoo A, et al. Worldwide ACE (I/D) polymorphism may affect COVID-19 recovery rate: an ecological meta-regression. Endocrine 2020;68:479-484.

23. Novelli A, Biancolella M, Borgiani P, et al. Analysis of ACE2 genetic variants in 131 Italian SARS-CoV2-positive patients. Hum Genomics 2020;14:29.

24. Gomez J, Albaiceta GM, Garcia-Clemente M, et al. Angiotensin-converting enzymes (ACE, ACE2) gene variants and COVID-19 outcome. Gene 2020;762:145102. 
25. Li Y, Li H, Zhou L. EZH2-mediated H3K27me3 inhibits ACE2 expression. Biochem Biophys Res Commun 2020;526:947-952.

26. Lambert DW, Clarke NE, Hooper NM, Turner AJ. Calmodulin interacts with angiotensin-converting enzyme-2 (ACE2) and inhibits shedding of its ectodomain. FEBS Lett 2008;582:385-90.

27. Saponaro F, Rutigliano G, Sestito S, et al. ACE2 in the Era of SARS-CoV-2: Controversies and Novel Perspectives. Front Mol Biosci 2020;7:588618.

28. Wang Y, Zhang L, Sang L, et al. Kinetics of viral load and antibody response in relation to COVID-19 severity. J Clin Invest 2020;130:5235-5244.

29. Liu Y, Yan LM, Wan L, et al. Viral dynamics in mild and severe cases of COVID-19. Lancet Infect Dis 2020;20:656-657.

30. Soy M, Keser G, Atagunduz P, Tabak F, Atagunduz I, Kayhan S. Cytokine storm in COVID-19: pathogenesis and overview of anti-inflammatory agents used in treatment. Clin Rheumatol 2020;39:20852094.

31. Liu Y, Liao W, Wan L, Xiang T, Zhang W. Correlation Between Relative Nasopharyngeal Virus RNA Load and Lymphocyte Count Disease Severity in Patients with COVID-19. Viral Immunol 2020 Apr 10. DOI: 10.1089/vim.2020.0062 [Epub ahead of print].

32. Peiris JS, Chu CM, Cheng VC, et al. Clinical progression and viral load in a community outbreak of coronavirus-associated SARS pneumonia: a prospective study. Lancet 2003;361:1767-72.

\section{Hosted file}

Tables.pdf available at https://authorea.com/users/401479/articles/513557-ace-i-d-and-ace2receptor-gene-rs2106809-rs2285666-polymorphisms-is-not-related-to-the-clinical-courseof-covid-19-a-case-study 